JLAB-THY-99-09

(version 2)

\title{
Flux Tube Zero-Point Motion, Hadronic Charge Radii, and Hybrid Meson Production Cross Sections
}

\author{
Nathan Isgur \\ Jefferson Lab, 12000 Jefferson Avenue, Newport News, Virginia, 23606
}

\begin{abstract}
Flux tube zero-point motion produces quark displacements transverse to the flux tube which make significant contributions to hadronic charge radii. In heavy quark systems, these contributions can be related by Bjorken's sum rule to the rates for semileptonic decay to hybrid mesons. This connection can be generalized to other leptoproduction processes, where transverse contributions to elastic form factor slopes are related to the cross sections for the production of the associated hybrid states. I identify the flux tube overlap integral responsible for these effects as the strong QCD analogue of the Sudakov form factor of perturbative QCD.
\end{abstract}




\section{INTRODUCTION}

The discovery and study of hybrid mesons is one of the most urgent tasks in strong interaction physics today, since their properties are a direct reflection of the mechanism of confinement. Theory [1,2] has provided compelling arguments from QCD that confinement occurs via the formation of a flux tube. In the simplest situation, corresponding to a long tube with fixed $Q$ and $\bar{Q}$ sources on its ends, a flux tube has a very simple vibrational spectrum corresponding to the excitation of transverse phonons in its string-like structure. The essential features of this gluonic spectrum are retained in the spectrum of real mesons with their flux tube excited (the hybrids), and consequently searches for hybrid mesons (especially those with tell-tale $J^{P C}$ exotic quantum numbers) are underway in many laboratories [3. 4 .

These searches will probably be technically difficult since the dominant final states resulting from the decays of the lightest hybrids are predicted [5, 6] to be very complex multiparticle states arising from the decay of intermediate $S+P$ decay products (where $S$ is an $\ell=0$ ground state meson and $P$ an $\ell=1$ excited state). In addition to their technical difficulty, hybrid meson searches have been considered difficult to interpret because hybrid meson production cross sections had only been estimated on very qualitative grounds [5]. The qualitative argument was frighteningly simple: a flux tube is a strongly interacting object, so it should be expected to be excited as readily as a quark. It followed that exclusive hybrid meson production cross sections should be comparable to exclusive ordinary meson production cross sections. This argument has recently been supported by a detailed Regge-theory-based calculation [7] for the electro- and photoproduction of the $J^{P C}=1^{-+}$ hybrid.

Using Bjorken's heavy quark sum rule [8.9], I present here an argument which relates the inclusive production cross section of hybrid mesons in heavy quark decay to a new contribution to the slope $\rho^{2}$ of the Isgur-Wise function [10]. This new contribution to elastic form factors is remarkably simple in character, but I can find no reference to it in the 
existing literature: zero-point oscillations of the confining flux tube will lead to transverse displacements of a meson's quarks from the interquark axis. These displacements contribute to the charge radius of a quark, making the elastic form factor fall faster than it would have otherwise. By Bjorken's sum rule (and its generalizations), this loss of rate from the elastic channel must be compensated by an inelastic channel. At the mechanical level, the loss of rate to the elastic channel occurs because the ground state wavefunctions of the flux tubes in the initial and final meson states do not overlap perfectly. It is then intuitively clear that the lost rate will appear in final states where the flux tube is not in its ground state, i.e., hybrid mesons. In what follows I will demonstrate this explicitly and show how the inclusive hybrid production cross sections are distributed into exclusive modes.

This connection between charge radii and hybrid production rates shows that the qualitative argument for the strength of hybrid meson cross sections presented in Ref. [5] was correct: hybrid production proceeds without suppression by any small parameters (nor any factors of $\left.1 / N_{c}\right)$. Bjorken's sum rule is only exact as a local duality in the heavy quark limit, but it can be generalized [11] via the operator product expansion (OPE) to an approximate global duality between hybrid meson and baryon electroproduction and a new contribution to the elastic charge radii of light hadrons.

\section{TRANSVERSE CONTRIBUTIONS TO HADRONIC CHARGE RADII}

In nonrelativistic quark potential models for $Q \bar{d}$ mesons, each quark develops a charge radius by making longitudinal excursions from the quark-antiquark center of mass along the interquark axis $\vec{r} \equiv \vec{r}_{\bar{d}}-\vec{r}_{Q}$. (I have employed a notation here that is suggestive of a heavy quark meson where $m_{Q} \gg m_{d}$ since Bjorken's sum rule applies exactly to this case, but I will keep open the possibility of arbitrary masses in most of the following.) In the nonrelativistic, valence approximation, $\vec{r}_{Q}=-\left(\frac{m_{d}}{m_{Q}+m_{d}}\right) \vec{r}$ so the squared charge radius of $Q$ is 


$$
r_{Q}^{2}=\left(\frac{m_{d}}{m_{Q}+m_{d}}\right)^{2}\left\langle r^{2}\right\rangle
$$

where $\left\langle r^{2}\right\rangle$ is the expectation value of the squared interquark separation in the meson wavefunction $\psi_{Q \bar{d}}(\vec{r})$. If the hadron in question has additional nonrelativistic constituents, as does a baryon, this relation is trivially modified with $\vec{r}$ interpreted as the relative coordinate of the center of mass of the constituents accompanying $Q$.

The flux tube between $Q$ and $\bar{d}$ is a relativistic object with an infinite number of degrees of freedom. For this reason the flux tube model is usually simplified by employing an adiabatic approximation which converts it into a quark potential model [1]. This approximation consists in first "nailing down" the ends of the flux tube and solving for its quantum states in the limit where it behaves like a thin piece of relativistic string with its purely transverse degrees of freedom. The resulting energies $E_{n}(r)$ ( $n$ defines the string's quantum state) are then used as (adiabatic or Born-Oppenheimer) effective potentials $V_{n}(r)$ on which meson spectroscopies are built: ordinary mesons are vibrational or rotational states built on the gluonic ground state effective potential $V_{0}(r)$, the lightest hybrids are built on the "one-phonon with wavelength $2 r$ " potential $V_{1}(r)$, etc. In this approximation, as in any nonrelativistic potential model, Eq. (11) continues to hold. However, for any finite quark mass, transverse fluctuations of the flux tube will lead to transverse excursions of $Q$ and a correction to this equation. In the next Section I derive the result

$$
r_{Q}^{2}=\left[\left(\frac{m_{d}}{m_{Q}+m_{d}}\right)^{2}+\frac{2 b}{\pi^{3} m_{Q}^{2}} \zeta(3)\right]\left\langle r^{2}\right\rangle
$$

or, in the heavy quark limit

$$
\rho^{2}=\frac{1}{3}\left[m_{d}^{2}+\frac{2 b}{\pi^{3}} \zeta(3)\right]\left\langle r^{2}\right\rangle
$$

where $\zeta(3) \equiv \sum_{p=1}^{\infty} \frac{1}{p^{3}} \simeq 1.20$. Note that, like the potential model term, the correction terms from flux-tube transverse oscillations are proportional to the mean square interquark distance. This occurs because the mass of the vibrating flux tube, to which $r_{Q \perp}^{2}$ is proportional, is $b r$. Using "canonical parameters" [1,12], Eq. (2) gives a huge $51 \%$ correction in light quark 
systems where $m_{Q}=m_{d}$; in heavy quark systems Eq. (3) gives a $13 \%$ correction. In light quark systems this missing transverse contribution could, along with now well-established relativistic corrections [13], be responsible for the infamous underprediction of charge radii in quark models.

The precise numerical values I have quoted for these corrections should not be taken too seriously, since the gluonic fields in ground state mesons are certainly not pure flux tubes. However, lattice QCD gives the remarkable result that $V_{0}(r)$ becomes linear at such small distances that much of even ground state wavefunctions may be flux tube dominated. This simple fact can only suggest that the qualitative character of the correction terms in Eqs. (2) and (3) will not be lost. However, I believe there is a compelling general physics case for such strong transverse corrections. Consider the $Q \bar{d}$ system immediately after the $Q$ makes a sudden transition from rest (as $m_{Q} \rightarrow \infty$, it has $\vec{v}=0$ ) to velocity $\vec{v}^{\prime}$. Independent of their geometry, by Gauss' law the color electric field lines in the neighborhood of $Q$ must immediately begin to follow it, but by causality those in the neighborhood of $\bar{d}$ must continue to point in the original direction of $Q$ for a time $T \geq r / c$. A "kink" will therefore be formed in the classical color electric field lines which will propagate from $Q$ to $\bar{d}$ with the period $T$. This classical kink in the color electric field will in turn manifest itself quantum mechanically in the excitation of gluonic degrees of freedom. (The preceeding description is the QCD analog of the textbook example presented in Ref. [14] for classical electromagnetism where the analogous excitation results in classical radiation and thus to the quantum emission of photons. In perturbative QCD the acceleration of $Q$ would result in the emission of gluons; in the strong QCD [15] regime of interest here the excitation is confined, making a hybrid meson.) Thus while flux tube dynamics provides a concrete model in which these transverse excitations and the associated transverse contributions to charge radii required by Bjorken's sum rule can be realized, it seems likely that the basic phenomenon is very general. In particular, even a Coulomb-like gluonic field would lead to similar effects of comparable strength (since $\alpha_{s} \sim 1$ in these systems). 
As a prelude to the next Section, I note that the sum $\zeta(3) \equiv \sum \frac{1}{p^{3}}$ in Eq. (3) is $\sim 80 \%$ saturated by its $p=1$ term. This strongly suggests what we will discover by explicit calculation below: hybrid production is dominantly in the lowest phonon mode. We will also see that a substantial fraction of this production goes into the mesonic ground state of the associated adiabatic surface $V_{1}(r)$.

\section{HYBRID EXCITATION AND BJORKEN'S SUM RULE}

To determine the contribution to $\left\langle\vec{r}_{Q}^{2}\right\rangle$ of the motion of the flux tube or to calculate the transition rates to the states in which the flux tube is excited, it is necessary to explicitly introduce the flux tube degrees of freedom into the description of hadrons. To do this, I adopt the method of Appendix A of Ref. [16] in which the flux tube is discretized into $N$ cells. In the adiabatic approximation in which the points $n=0$ and $N+1$ are fixed on static

quarks and $n=1,2, \ldots N$ have transverse degrees of freedom $\vec{y}_{n}$, the state of the flux tube can be written in terms of a complete set of transverse eigenstates

$$
|\vec{y}\rangle=\left|\vec{y}_{1} \vec{y}_{2} \cdots \vec{y}_{N}\right\rangle
$$

Alternatively, and more conveniently, the Fourier coefficients

$$
\vec{a}_{p} \equiv \sum_{n=1}^{N} \vec{y}_{n} \sqrt{\frac{2}{N+1}} \sin \frac{p \pi}{N+1} n
$$

can be introduced and the state of the flux tube can be written in terms of a complete set of Fourier amplitudes

$$
|\vec{a}\rangle=\left|\vec{a}_{1} \vec{a}_{2} \cdots \vec{a}_{N}\right\rangle
$$

The classical Lagrangian for this system in the small oscillations approximation is

$$
L\left(\vec{y}, \frac{d \vec{y}}{d t}\right)=b a \sum_{n=0}^{N}\left[\frac{1}{2}\left(\frac{d \vec{y}_{n}}{d t}\right)^{2}-\frac{1}{2 a^{2}}\left(\vec{y}_{n+1}-\vec{y}_{n}\right)^{2}\right]
$$

where $b$ is the string tension and $a$ is the length of each cell. Transforming to the Fourier coefficients gives 


$$
L\left(\vec{a}, \frac{d \vec{a}}{d t}\right)=b a \sum_{p=1}^{N}\left[\frac{1}{2}\left(\frac{d \vec{a}_{p}}{d t}\right)^{2}-\frac{1}{2} \omega_{p}^{2} \vec{a}_{p}^{2}\right]
$$

where

$$
\omega_{p} \equiv \frac{2}{a} \sin \frac{\pi p}{2(N+1)}
$$

In the Fourier basis the eigenstates can be labelled by occupation numbers $n_{p}^{\alpha}$, the number of "phonons" in mode $p$ with transverse polarization $\alpha=1$ or 2 :

$$
|n\rangle=\left|n_{1}^{1} n_{1}^{2} n_{2}^{1} n_{2}^{2} \cdots n_{N}^{1} n_{N}^{2}\right\rangle
$$

with $E_{n}=\sum_{p=1}^{N}\left(n_{p}^{1}+n_{p}^{2}+1\right) \omega_{p}$ and a wavefunction

$$
\chi_{n}(\vec{a}) \equiv\langle\vec{a} \mid n\rangle=\chi_{n_{1}}\left(a_{1}^{1}\right) \chi_{n_{1}}\left(a_{1}^{2}\right) \cdots \chi_{n_{N}}\left(a_{N}^{1}\right) \chi_{n_{N}}\left(a_{N}^{2}\right)
$$

where

$$
\chi_{0_{p}}\left(a_{p}^{\alpha}\right)=\frac{\alpha_{p}^{1 / 2}}{\pi^{1 / 4}} \exp \left[-\frac{1}{2} \alpha_{p}^{2}\left(a_{p}^{\alpha}\right)^{2}\right]
$$

and

$$
\chi_{1_{p}}\left(a_{p}^{\alpha}\right)=\sqrt{2} \alpha_{p} a_{p}^{\alpha} \psi_{0_{p}^{\alpha}}\left(a_{p}^{\alpha}\right)
$$

are the wavefunctions we will need here. Note that

$$
\alpha_{p}^{2}=2 b \sin \frac{\pi p}{2(N+1)}
$$

follows from Eq. (8).

To study the effects of the flux tube on form factors, we must go beyond the static approximation to consider the normal modes of the flux tube-quark system. For large quark masses $m_{d}, m_{Q} \gg b r$, the transverse oscillations of frequency $\omega_{p}=\pi p / r$ are fast relative to the nearly static longitudinal motion, and so these normal modes may be considered to be at fixed $r$. (In the low-lying states of a linear potential, the ratio of these two frequencies is $\left(\mu_{Q \bar{d}}^{2} / b\right)^{\frac{1}{3}}$, where $\mu_{Q \bar{d}}$ is the $Q \bar{d}$ reduced mass.) In these circumstances one can easily show 
using conservation of the position of the center of mass and of orbital angular momentum about the center of mass that the mode $p$ with amplitude $\vec{a}_{p}$ involves transverse excursions of the $Q$ and $\bar{d}$ quarks given by

$$
\begin{aligned}
& \vec{r}_{Q_{\perp}}=-\frac{b r}{\pi p m_{Q}} \sqrt{\frac{2}{N+1}} \vec{a}_{p} \\
& \vec{r}_{\bar{d}_{\perp}}=(-1)^{p} \frac{b r}{\pi p m_{d}} \sqrt{\frac{2}{N+1}} \vec{a}_{p} .
\end{aligned}
$$

We are now in a position to write down the full flux tube model state vectors of a $Q \bar{d}$ meson located at center of mass position $\vec{R}$. The $n^{\text {th }}$ normal $Q \bar{d}$ meson $M_{n}^{(0)}$ built on the adiabatic potential $V_{0}(r)$ corresponding to the flux tube ground state is

$$
\begin{aligned}
\left|M_{n}^{(0)}(\vec{R})\right\rangle= & \int d^{3} r \int d^{2} a_{1} \cdots \int d^{2} a_{N} \psi_{n}^{(0)}(\vec{r}) \chi_{0_{1}}\left(a_{1}^{1}\right) \chi_{0_{1}}\left(a_{1}^{2}\right) \cdots \\
& \chi_{0_{N}}\left(a_{N}^{1}\right) \chi_{0_{N}}\left(a_{N}^{2}\right) \mid Q\left(\vec{R}-\frac{m_{d}+\frac{1}{2} b r}{m_{Q}+m_{d}+b r} \vec{r}-\frac{b r}{\pi m_{Q}} \sqrt{\frac{2}{N+1}} \sum_{p} \frac{1}{p} \vec{a}_{p}\right) ; \\
& \left.\vec{a}_{1} \vec{a}_{2} \cdots \vec{a}_{N} ; d\left(\vec{R}+\frac{m_{Q}+\frac{1}{2} b r}{m_{Q}+m_{d}+b r} \vec{r}+\frac{b r}{\pi m_{d}} \sqrt{\frac{2}{N+1}} \sum_{p} \frac{(-1)^{p}}{p} \vec{a}_{p}\right)\right\rangle
\end{aligned}
$$

while the $n^{\text {th }}$ hybrid $Q \bar{d}$ meson built on the potential $V_{1}(r)$ corresponding to the excitation of one phonon in the lowest modes $p=1, \alpha=1$, and $p=1, \alpha=2$ are

$$
\begin{aligned}
\left|H_{n \ell m}^{(1 \pm)}(\vec{R})\right\rangle= & \int d^{3} r \int d^{2} a_{1} \cdots \int d^{2} a_{N} \psi_{n \ell m}^{(1 \pm)}(\vec{r}) \alpha_{1}\left(a_{1}^{1} \pm i a_{1}^{2}\right) \chi_{0_{1}}\left(a_{1}^{1}\right) \chi_{0_{1}}\left(a_{1}^{2}\right) \cdots \\
& \chi_{0_{N}}\left(a_{N}^{1}\right) \chi_{0_{N}}\left(a_{N}^{2}\right) \mid Q\left(\vec{R}-\frac{m_{d}+\frac{1}{2} b r}{m_{Q}+m_{d}+b r} \vec{r}-\frac{b r}{\pi m_{Q}} \sqrt{\frac{2}{N+1}} \sum_{p} \frac{1}{p} \vec{a}_{p}\right) ; \\
& \left.\vec{a}_{1} \vec{a}_{2} \cdots \vec{a}_{N} ; d\left(\vec{R}+\frac{m_{Q}+\frac{1}{2} b r}{m_{Q}+m_{d}+b r} \vec{r}+\frac{b r}{\pi m_{d}} \sqrt{\frac{2}{N+1}} \sum_{p} \frac{(-1)^{p}}{p} \vec{a}_{p}\right)\right\rangle .
\end{aligned}
$$

In these expressions the quarks $Q$ and $\bar{d}$ are in position eigenstates which include their equilibrium separation $\vec{r}$ and their excursions due to Eqs. (15) and (16), while the flux tube state is described in a normal mode expansion with respect to its degrees of freedom $a_{p}^{\alpha}$ transverse to $\vec{r}$. Good orbital angular momentum states in the hybrid sector have been formed by using circularly polarized phonon states built out of $\alpha=1$ and 2 with angular momenta $\pm \hbar$ around the axis $\vec{r} \leftrightarrow(r \theta \phi)$ so that, for example 


$$
\begin{aligned}
\psi_{n 11}^{(1+)} & =\sqrt{\frac{3}{4 \pi}} \tilde{\psi}_{H_{n}^{(1)}}(r)\left[\frac{1+\cos \theta}{2}\right] \\
\psi_{n 10}^{(1+)} & =\sqrt{\frac{3}{4 \pi}} \tilde{\psi}_{H_{n}^{(1)}}(r)\left[\sqrt{\frac{1}{2}} \sin \theta\right] e^{-i \phi} \\
\psi_{n 1-1}^{(1+)} & =\sqrt{\frac{3}{4 \pi}} \tilde{\psi}_{H_{n}^{(1)}}(r)\left[\frac{1-\cos \theta}{2}\right] e^{-2 i \phi}
\end{aligned}
$$

where $\tilde{\psi}_{H_{n}^{(1)}}(r)$ is normalized to $\int d r r^{2}\left|\tilde{\psi}_{H_{n}^{(1)}}(r)\right|^{2}=1$. Note that the normal mode expansion is with respect to body-fixed unit vectors which can be expanded as

$$
\begin{aligned}
& \hat{e}_{1}(\vec{r})=\left[\left(\frac{1+\cos \theta}{2}\right)-\left(\frac{1-\cos \theta}{2}\right) \cos 2 \phi\right] \hat{x}-\left(\frac{1-\cos \theta}{2}\right) \sin 2 \phi \hat{y}-\sin \theta \cos \phi \hat{z} \\
& \hat{e}_{2}(\vec{r})=-\left(\frac{1-\cos \theta}{2}\right) \sin 2 \phi \hat{x}+\left[\left(\frac{1+\cos \theta}{2}\right)+\left(\frac{1-\cos \theta}{2}\right) \cos 2 \phi\right] \hat{y}-\sin \theta \sin \phi \hat{z}
\end{aligned}
$$

relative to the space-fixed coordinate axes $\hat{x}, \hat{y}, \hat{z}$. These expressions follow from a rigid rotation of the flux tube from $r \hat{z}$ to $\vec{r}$ in the $(r \hat{z}, \vec{r})$ plane. Finally, before proceeding to calculate form factors, we form total momentum eigenstates

$$
|\vec{P}\rangle=\int d^{3} R \frac{e^{i \vec{P} \cdot \vec{R}}}{(2 \pi)^{3 / 2}}|\vec{R}\rangle
$$

where $|\vec{R}\rangle$ is either set of position eigenstates.

I begin by using this machinery to calculate the elastic form factor for scattering the pseudoscalar ground state $P \equiv M_{0}^{(0)}$ from momentum $-\vec{P}_{c m} / 2$ to $+\vec{P}_{c m} / 2$ via the current $\bar{Q} \Gamma Q$. Since by heavy quark symmetry the Isgur-Wise function is to leading order independent of both the heavy quark spin and the Dirac structure of the current, I use scalar quarks $Q$ and the current $Q^{\dagger} Q$. With the standard heavy quark velocity transfer variable $w-1=P_{c m}^{2} / 2 m_{Q}^{2}=V_{c m}^{2} / 2$, we then have as $m_{Q} \rightarrow \infty$

$$
\begin{aligned}
\xi(w) \equiv & (2 \pi)^{3}\left\langle P\left(+\frac{\vec{P}_{c m}}{2}\right)\left|Q^{\dagger}(0) Q(0)\right| P\left(-\frac{\vec{P}_{c m}}{2}\right)\right\rangle \\
= & \int d^{3} r \int d^{2} a_{1} \cdots \int d^{2} a_{N} e^{-i \vec{V}_{c m} \cdot\left[\left(m_{d}+\frac{1}{2} b r\right) \vec{r}+\frac{b r}{\pi} \sqrt{\frac{2}{N+1}} \sum_{p} \frac{1}{p} \vec{a}_{p}\right]} \\
& \left|\psi_{0}^{(0)}(\vec{r})\right|^{2}\left|\chi_{0_{1}}\left(a_{1}^{1}\right)\right|^{2}\left|\chi_{0_{1}}\left(a_{1}^{2}\right)\right|^{2} \cdots\left|\chi_{0_{N}}\left(a_{N}^{1}\right)\right|^{2}\left|\chi_{0_{N}}\left(a_{N}^{2}\right)\right|^{2} .
\end{aligned}
$$

Since we are interested in calculating the slope $\rho^{2}$ in

$$
\xi(w) \simeq 1-\rho^{2}(w-1)+\cdots
$$


we can expand the exponential to order $V_{c m}^{2}$ to get

$$
\begin{aligned}
\xi(w) \simeq & 1-\frac{1}{2} V_{c m}^{i} V_{c m}^{j} \int d^{3} r \int d^{2} a_{1} \cdots \int d^{2} a_{N}\left[\left(m_{d}+\frac{1}{2} b r\right)^{2} r^{i} r^{j}+\frac{2 b^{2} r^{2}}{\pi^{2}(N+1)} \sum_{p \alpha} \frac{\left(a_{p}^{\alpha}\right)^{2}}{p^{2}} \hat{e}_{\alpha}^{i} \hat{e}_{\alpha}^{j}\right] \\
& \left|\psi_{0}^{(0)}(\vec{r})\right|^{2}\left|\chi_{0_{1}}\left(a_{1}^{1}\right)\right|^{2}\left|\chi_{0_{1}}\left(a_{1}^{2}\right)\right|^{2} \cdots\left|\chi_{0_{N}}\left(a_{N}^{1}\right)\right|^{2}\left|\chi_{0_{N}}\left(a_{N}^{2}\right)\right|^{2} \\
\simeq & 1-\frac{\left\langle\left(m_{d}+\frac{1}{2} b r\right)^{2} r^{2}\right\rangle}{3}(w-1)-\rho_{\text {transverse }}^{2}(w-1) \cdots
\end{aligned}
$$

where

$$
\begin{array}{r}
\rho_{\text {transverse }}^{2}(w-1) \equiv \frac{1}{2} V_{c m}^{i} V_{c m}^{j} \int d^{3} r \int d^{2} a_{1} \cdots \int d^{2} a_{N}\left[\frac{2 b^{2} r^{2}}{\pi^{2}(N+1)} \sum_{p \alpha} \frac{\left(a_{p}^{\alpha}\right)^{2}}{p^{2}} \hat{e}_{\alpha}^{i} \hat{e}_{\alpha}^{j}\right] \\
\left|\psi_{0}^{(0)}(\vec{r})\right|^{2}\left|\chi_{0_{1}}\left(a_{1}^{1}\right)\right|^{2}\left|\chi_{0_{1}}\left(a_{1}^{2}\right)\right|^{2} \cdots\left|\chi_{0_{N}}\left(a_{N}^{1}\right)\right|^{2}\left|\chi_{0_{N}}\left(a_{N}^{2}\right)\right|^{2}
\end{array}
$$

is the new contribution to $\rho^{2}$ from flux tube transverse oscillations. (Note that Eq. (29) indicates that the ordinary longitudinal contribution $m_{d}^{2}\left\langle r^{2}\right\rangle / 3$ should be supplemented with a term which includes the flux tube contribution to the center of mass. While keeping $\frac{1}{2} b r$ in comparison with $m_{d}$ in this term is not obviously consistent with the $\mathrm{br} / \mathrm{m}_{d}$ expansion we used to justify the adiabatic treatment of transverse oscillations relative to longitudinal motion, I believe this $i s$ the next term in the expansion of the longitudinal motion of $\vec{r}_{Q}$. I nevertheless refrain from interpreting the extra $\frac{1}{2} b r$ term as a "new" contribution to charge radii since it seems likely that the constituent quark mass of quark models has been chosen to at least include a part of its effect. Thus with $m_{d}$ interpreted as the light constituent quark mass, Eq. (21) rather than (29) should be used.) With Eqs. (23), the required angular averages may be done to obtain

$$
\rho_{\text {transverse }}^{2}=\frac{2 b\left\langle r^{2}\right\rangle}{3 \pi^{3}} \zeta(3)
$$

as claimed in Eq.(3). The previously quoted result (2) for the transverse correction to a normal charge radius follows by exactly parallel steps to those just outlined but avoiding the use of the approximation $\vec{P}_{c m} \simeq m_{Q} \vec{V}_{c m}$.

Both (2) and (3) are simply the results of averaging over the zero-point fluctuations of the $\vec{a}_{p}$, and could perhaps be obtained more simply if $\rho_{\text {transverse }}^{2}$ and $r_{\text {transverse }}^{2}$ were our only 
interests. However, with this machinery, we can do much more. For example, Eq. (28) gives us the complete function $\xi(w)$. The new flux tube contribution to this function represents the probability that, in suffering the action of the current $Q^{\dagger} Q$, the gluonic degrees of freedom were not excited. At large recoils, probing short distances, these flux tube excitations will become gluon jet events of perturbative QCD, and the analogous probability of not emitting gluon radiation will be the Sudakov form factor [17]. In heavy quark systems this probability appears in the (scale-dependent) contribution to $\rho^{2}$

$$
\Delta \rho_{\text {pert }}^{2} \simeq \frac{16}{81} \ln \left[\frac{\alpha_{s}(\mu)}{\alpha_{s}\left(m_{c}\right)}\right]
$$

which arises from the velocity-dependent anomalous dimension of the heavy quark effective theory matching coefficients [18,17]. If one assumes that $\mu$ can be taken as low as the quark model scale, then $\Delta \rho_{\text {pert }}^{2} \simeq 0.13 \pm 0.05$ [12], quite comparable to the strong QCD contribution to $\rho^{2}$ derived here. While we will not display or further discuss the full function $\xi(w)$ here, the machinery we have introduced is also required to explicitly compute the rates of excitation of the hybrid states that compensate for $\rho_{\text {transverse }}^{2}$ to satisfy Bjorken's sum rule. We now turn to that task.

The inelastic form factor for the production of the hybrid $H_{n \ell m}^{(1 \pm)}$ is

$$
\begin{aligned}
\tau_{ \pm n \ell m}(w) \equiv & (2 \pi)^{3}\left\langle H_{n \ell m}^{(1 \pm)}\left(+\frac{\vec{P}_{c m}}{2}\right)\left|Q^{\dagger}(0) Q(0)\right| P\left(-\frac{\vec{P}_{c m}}{2}\right)\right\rangle \\
= & \left.\int d^{3} r \int d^{2} a_{1} \cdots \int d^{2} a_{N} e^{-i \vec{V}_{c m} \cdot\left[\left(m_{d}+\frac{1}{2} b r\right) \vec{r}+\frac{b r}{\pi}\right.} \sqrt{\frac{2}{N+1}} \sum_{p} \frac{1}{p} \vec{a}_{p}\right] \\
& \psi_{n \ell m}^{(1 \pm)}(\vec{r})^{*} \psi_{0}^{(0)}(\vec{r}) \alpha_{1}\left(a_{1}^{1} \mp i a_{1}^{2}\right)\left|\chi_{0_{1}}\left(a_{1}^{1}\right)\right|^{2}\left|\chi_{0_{1}}\left(a_{1}^{2}\right)\right|^{2} \cdots\left|\chi_{0_{N}}\left(a_{N}^{1}\right)\right|^{2}\left|\chi_{0_{N}}\left(a_{N}^{2}\right)\right|^{2} .
\end{aligned}
$$

To order $(w-1)$ this integral is nonzero only through the term $\vec{V}_{c m} \cdot \vec{a}_{1}$ in the expansion of the exponential, so we have

$$
\begin{aligned}
\tau_{ \pm n \ell m}(w) \simeq & -\frac{i b \alpha_{1}}{\pi} \sqrt{\frac{2}{N+1}} \int d^{3} r \int d^{2} a_{1}\left[\vec{V}_{c m} \cdot \vec{a}_{1}\right] \\
& \psi_{n \ell m}^{(1 \pm)}(\vec{r})^{*} \psi_{0}^{(0)}(\vec{r}) r\left(a_{1}^{1} \mp i a_{1}^{2}\right)\left|\chi_{0_{1}}\left(a_{1}^{1}\right)\right|^{2}\left|\chi_{0_{1}}\left(a_{1}^{2}\right)\right|^{2} \\
\simeq & -i \sqrt{\frac{b}{2 \pi^{3}}} \int d^{3} r \psi_{n \ell m}^{(1 \pm)}(\vec{r})^{*} \psi_{0}^{(0)}(\vec{r}) r \\
& {\left[\left(\frac{1+\cos \theta}{2}\right) V_{c m-}-\sin \theta e^{-i \phi} V_{c m z}-\left(\frac{1-\cos \theta}{2}\right) e^{-2 i \phi} V_{c m+}\right] }
\end{aligned}
$$


which implies that only $\ell=1$ states are needed to saturate Bjorken's sum rule [9]. Using Eqs. (19)-(21), it follows that

$$
\tau_{ \pm n \ell m}(w)=\tau_{n}(w) \delta_{\ell 1} V_{c m m}^{*}
$$

where $V_{c m+} \equiv-\left(V_{c m x}+i V_{c m y}\right), V_{c m 0} \equiv \sqrt{2} V_{c m z}$, and $V_{c m-} \equiv-\left(V_{c m x}-i V_{c m y}\right)$ and where

$$
\tau_{n}(w)=-i \sqrt{\frac{b}{6 \pi^{3} m_{d}^{2}}} \int d r r^{3} \tilde{\psi}_{H_{n}^{(1)}}(r)^{*} \tilde{\psi}_{P}(r)
$$

with $\tilde{\psi}_{H_{n}^{(1)}}(r)$ and $\tilde{\psi}_{P}(r)$ the pure radial parts of the wavefunctions $\psi_{n \ell m}^{(1 \pm)}(\vec{r})$ and $\psi_{0}^{(0)}(\vec{r})$, respectively, orthonormalized in their sectors to $\int d r r^{2} \tilde{\psi}_{m}(r)^{*} \tilde{\psi}_{n}(r)=\delta_{m n}$. The total rate of transitions to both \pm "helicity" states and all magnetic substates in units of the elastic rate is therefore

$$
R_{H_{n}^{(1)}}=\frac{4 b}{3 \pi^{3}}(w-1)\left|\int d r r^{3} \tilde{\psi}_{H_{n}^{(1)}}(r)^{*} \tilde{\psi}_{P}(r)\right|^{2}
$$

It follows that

$$
\sum_{n} R_{H_{n}^{(1)}}=\frac{4 b\left\langle r^{2}\right\rangle}{3 \pi^{3}}(w-1)
$$

since the $\psi_{H_{n}^{(1)}}(r)$ are a complete set of orthonormal states over $r$. On the other hand, the loss of rate from the elastic channel due to transverse modes is (in units of the elastic rate) $2 \rho_{\text {transverse }}^{2}$, so we see on examining Eq. (31) that the total rate into the $p=1$ modes exactly compensates the $p=1$ term in the loss of elastic rate.

Note that the rate to the hybrids $H_{n}^{(1)}$ is not highly fragmented. Using "canonical" parameters [1], it is straightforward to calculate the ratio

$$
r \equiv \frac{\left|\int d r r^{3} \tilde{\psi}_{H_{0}^{(1)}}(r)^{*} \tilde{\psi}_{P}(r)\right|^{2}}{\left\langle r^{2}\right\rangle}
$$

which determines the fractional rate to the lowest-lying $p=1$ hybrid mesons. This ratio is about $40 \%$, so a substantial fraction of the total hybrid production will go into the lightest states. Unfortunately, these heavy-light states cannot have the $J^{P C}$ exotic quantum 
numbers which create "smoking gun" signals for exotics in the $u \bar{u}, u \bar{d}, d \bar{d}, s \bar{s}, c \bar{c}$, ... sectors. This fact will make their observation (and in particular distinguishing them from normal $c \bar{d}$ resonances) difficult in $\bar{B}$ decay. Moreover, their expected $3 \mathrm{GeV}$ masses will substantially reduce the phase space available to their semileptonic decay, suppressing their production rate in $\bar{B}$ decay well below that expected in the heavy quark limit, and thereby violating duality 19.

\section{GENERALIZATIONS AND CONCLUSIONS}

In this paper I have focussed on heavy quark semileptonic decay where exact duality via Bjorken's sum rule allowed the derivation of a precise relation between transverse contributions to the position $\vec{r}_{Q}$ of the heavy quark and the rate of hybrid meson production. The most important result of this examination was the observation that since

$$
\frac{\left\langle r_{Q \perp}^{2}\right\rangle}{\left\langle r_{Q}^{2}\right\rangle}=\frac{2 b}{\pi^{3} m_{d}^{2}} \zeta(3)
$$

is a pure number of order unity in QCD $\left(m_{d}\right.$ is the constituent quark mass and so is proportional to $b^{1 / 2}$ ), hybrid meson production in heavy quark semileptonic decay is unsuppressed by any small parameters.

While the lack of $J^{P C}$ exotic signals and phase space suppression may make the search for these states difficult in semileptonic $\bar{B}$ decay, the generalization of these rigorously derived results should provide comfort and guidance to experimentalists planning to search for hybrids in other processes. Consider first the $\bar{B}$ system itself. The factorizing components [20] of $\bar{B}$ hadronic decays to charm, e.g. $\bar{B} \rightarrow \pi^{-} X_{c}$ and $\bar{B} \rightarrow \rho^{-} X_{c}$, should populate hybrid states $X_{c}$ in the same proportions as in semileptonic decay. The analogous statement holds for the rare factorizing $\bar{B}$ hadronic decays induced by an underlying $b \rightarrow u$ transition, e.g., $\bar{B} \rightarrow \pi^{-} X_{u}$ and $\bar{B} \rightarrow \rho^{-} X_{u}$, where in $B^{-}$decay one would have the advantage of producing

$J^{P C}$ exotic hybrid mesons. (In contrast, nonfactorizing processes like $\bar{B} \rightarrow \bar{K} X_{c \bar{c}}$ cannot be expected to copiously produce $c \bar{c}$ hybrids [21] because the $c \bar{c}$ pair is being created by a 
local effective operator.) The next most immediate generalization is perhaps to ordinary electroproduction. In this process there is an analogue of Bjorken's sum rule [11] and thus of all of our results. In particular, $e p \rightarrow e^{\prime} X$ should produce ordinary $N^{*}$ 's and hybrid baryons with comparable cross sections once the threshold for hybrid baryons is crossed. By the same token, meson electroproduction by electroexcitation of an exchanged meson (the Sullivan processes [22]) should readily produce hybrid mesons. (Recall that for light quarks the hybrid fraction is four times larger than in the heavy quark limit.) While more difficult to study experimentally, the advantage of meson electroproduction over baryon electroproduction is that the former process will produce $J^{P C}$ exotic signals while the latter cannot.

I am convinced that there are also generalizations of the results of this paper much farther afield than electroproduction. Consider, for example, forward photoproduction. In this process strong interactions convert the virtual vector meson component of the photon into excited mesons. If these interactions are dominantly with the quarks in the virtual vector mesons, they will induce dynamical processes very similar to those described here. If they are dominantly with the flux tube, then they can directly excite one of its phonon modes, and one can expect such direct hybrid production to be even stronger than quark-recoil-induced production. A very similar generalization applies to forward meson hadroproduction. The main difference between hadroproduction and photoproduction is that the quark spin of unity in the latter process is more likely to lead to the desirable $J^{P C}$ exotic hybrids [5].

In summary, I have identified an important new transverse contribution to hadronic charge radii from flux tube zero-point motion. Since the transverse quark motion has the universal QCD scale $b^{\frac{1}{2}}$, its contribution to hadronic charge radii is comparable to that from longitudinal quark motion. There are consequently substantial corrections to potential model estimates of charge radii. Given that the transverse and longitudinal radii are comparable, it follows by Bjorken's sum rule that hybrid production in the heavy quark limit of $Q_{1} \rightarrow Q_{2} \ell \bar{\nu}_{\ell}$ semileptonic decay will be comparable to ordinary resonance production. I have explicitly discussed the generalization of this result to a broad range of possible hy- 
brid production processes, and argued that hybrid searches underway and in planning can safely assume that their hybrid cross sections, unless forbidden by general selection rules, will indeed be comparable to those of ordinary mesons with the same phase space factors.

\section{REFERENCES}

[1] N. Isgur and J. Paton, Phys. Rev. D 31, 2910 (1985).

[2] For a recent review of both lattice and analytic approaches to flux tubes, see "Quark Confinement and the Hadron Spectrum II", ed. N. Brambilla and G.M. Prosperi (World Scientific, Singapore, 1997); and "Confinement III", Proceedings of the Third International Conference on Quark Confinement and the Hadron Spectrum, ed. N. Isgur (World Scientific, Singapore, in press).

[3] E852 Collaboration, D.R. Thompson et al., Phys. Rev. Lett. 79, 1630 (1997).

[4] G.T. Condo et al., Phys. Rev. D43, 2787 (1991); The Omega Photon Collaboration, M. Atkinson et al., Z. Phys. C34, 157 (1987); G.R. Blackett et al., hep-ex/9708032; The CLAS Collaboration, G. Adams et al., "Exotic Meson Spectroscopy with CLAS", CEBAF proposal E-94-121; I Aznauryan et al., "Search for $J^{P C}=1^{-+}$Exotic Mesons ...", CEBAF proposal E-94-118.

[5] N. Isgur, R. Kokoski, and J. Paton, Phys. Rev. Lett. 54, 869 (1985).

[6] F.E. Close and P.R. Page, Nucl. Phys. B443, 233 (1995); Phys. Rev. D52, 1706 (1995); T. Barnes, F.E. Close, and E.S. Swanson, Phys. Rev. D52, 5242 (1995).

[7] A. Afanasev and P.R. Page, Phys. Rev. D57, 6771 (1998).

[8] J.D. Bjorken, in Proceedings of the $4^{\text {th }}$ Rencontre de Physique de la Vallee d'Aoste, La Thuile, Italy, 1990, ed. M. Greco (Editions Frontieres, Gif-sur-Yvette, France, 1990); J. D. Bjorken, 
Recent Developments in Heavy Flavor Theory, in Proceedings of the XXVth International Conference on High Energy Physics, Singapore, (World Scientific, Singapore, 1992).

[9] N. Isgur and M.B. Wise, Phys. Rev. D43, 819 (1991).

[10] For the original papers see N. Isgur and M.B. Wise, Phys. Lett. B232, 113 (1989); Phys. Lett. B237, 527 (1990). For an overview of heavy quark symmetry see N. Isgur and M.B. Wise, "Heavy Quark Symmetry" in B Decays, ed. S. Stone (World Scientific, Singapore, 1991), p. 158, and in "Heavy Flavors", ed. A.J. Buras and M. Lindner (World Scientific, Singapore, 1992), p. 234.

[11] N. Cabibbo and L. Radicati, Phys. Lett. 19, 697 (1966).

[12] D. Scora and N. Isgur, Phys. Rev. D 52, 2783 (1995) present an updated version of the ISGW model of N. Isgur, D. Scora, B. Grinstein, and M. B. Wise, Phys. Rev. D 39, 799 (1989) called ISGW2.

[13] See, e.g., the discussion of relativistic corrections to charge radii in Ref. [12] and the sources cited therein.

[14] Edward M. Purcell, "Electricity and Magnetism" (McGraw-Hill, New York, 1964), pp. 163-167.

[15] F.E. Close, private communication, cogently advocates using the term "strong QCD" instead of the negative "nonperturbative QCD".

[16] R. Kokoski and N. Isgur, Phys. Rev. D 35, 907 (1987).

[17] For the connection between the Sudakov form factor, the heavy quark velocity-dependent anomalous dimension [18], and kinks in Wilson lines, see G.P. Korchemsky and A.V. Radyushkin, Phys. Lett. B279, 359 (1992); for work on each of these topics, see the references quoted therein.

[18] A.F. Falk, H. Georgi, B. Grinstein and M.B. Wise, Nucl. Phys. B343, 1 (1990).

[19] N. Isgur, Phys. Lett. B448, 111 (1999). 
[20] M.J. Dugan and B. Grinstein, Phys. Lett. B257, 399 (1991); C. Reader and N. Isgur, Phys. Rev. D47, 1007 (1993); for a review see M. Neubert, V. Rieckert, B. Stech, and Q.P. Xu, in "Heavy Flavors", ed. A.J. Buras and M. Lindner (World Scientific, Singapore, 1992), p. 286.

[21] F.E. Close, I. Dunietz, P.R. Page, S. Veseli, and H. Yamamoto, Phys. Rev. D57, 5653 (1998); G. Chiladze, A.F. Falk, and A.A. Petrov, Phys. Rev. D58, 034013 (1999). I am grateful to Philip Page for bringing these papers to my attention.

[22] J.D. Sullivan, Phys. Rev. D5, 1732 (1972). 